\title{
On the age-independent publication index
}

\author{
Keshra Sangwal
}

Received: 4 January 2012/Published online: 20 January 2012

(C) The Author(s) 2012. This article is published with open access at Springerlink.com

\begin{abstract}
It is shown that the age-independent index based on $h$-type index per decade, called hereafter an $\alpha$ index instead of the $a$ index, suggested by Kosmulski (Journal of Informetrics 3, 341-347, 2009) and Abt (Scientometrics 2012) is related to the square-root of the ratio of citation acceleration $a$ to the Hirsch constant $A$.
\end{abstract}

Keywords Age-independent index $\alpha$. Citation acceleration $a \cdot$ Hirsch index $h$. Hirsch constant $A$

It is well known (Hirsch 2005; Anderson et al. 2008; Kosmulski 2009; Abt 2011) that the Hirsch index $h$ of an author increases with his/her publication duration $t$. Therefore, the $h$ index cannot be used to compare the scientific output of two authors working in a particular field for different durations. It is observed that: (1) if the $h$ index of an author is divided by the number of decades since the publication of his/her first paper, one obtains a statistically constant index which is independent of his/her age (Kosmulski 2009; Abt 2011), and (2) the accuracy of this index is the same as that of the $h$ index (Abt 2011). These authors proposed that one can compare the publication activity of authors of different ages using this age-independent index.

In this communication it is shown that the age-independent index, called hereafter an $\alpha$ index instead of the $a$ index and $h$-type index per decade (hpd index) suggested by Abt and Kosmulski, respectively, is related to the square-root of the ratio of citation acceleration $a$ to the Hirsch constant $A$. The citation acceleration $a$ and the Hirsch constant $A$ are related to the total number of citations $L$ given by Eqs. 2 and 1, respectively. We have analyzed here the citation data of the age-independent $\alpha$ index for six scientists elected to membership of the Royal Society in 2006, randomly chosen by Anderson et al. (2008), and 12 astronomers considered by Abt (2011). These data are given in Tables 1 and 2, which include the total number $N$ of papers, the total publication duration $t$, the total number $L$ of

K. Sangwal $(\bowtie)$

Department of Applied Physics, Lublin University of Technology, ul. Nadbystrzycka 38,

20-618 Lublin, Poland

e-mail: k.sangwal@pollub.pl 
Table 1 Citation parameters of selected authors of Anderson et al. (2008)

\begin{tabular}{lrrlllrl}
\hline Author & $N(t)$ & \multicolumn{1}{l}{$L$} & \multicolumn{1}{l}{$h$} & \multicolumn{1}{l}{$a$} & \multicolumn{1}{l}{$A$} & \multicolumn{1}{l}{$10 b^{*}$} \\
\hline D. Badford & $78(20)$ & 6281 & 44 & $20.0 \pm 6.0$ & 15.70 & 3.24 & $22.0(18.01 \pm 0.53)$ \\
A.D. Becke & $55(28)$ & 40094 & 35 & $14.67 \pm 1.45$ & 51.14 & 32.73 & $12.5(15.79 \pm 0.26)$ \\
M. Lockwood & $176(25)$ & 5101 & 39 & $12.48 \pm 4.08$ & 8.16 & 3.35 & $15.6(11.62 \pm 0.49)$ \\
R.J. Jackson & $79(36)$ & 10778 & 44 & $11.92 \pm 2.04$ & 8.32 & 5.57 & $12.22(11.12 \pm 0.18)$ \\
M.R.E. Proctor & $89(31)$ & 2356 & 26 & $8.33 \pm 0.88$ & 2.45 & 3.48 & $8.39(8.70 \pm 0.44)$ \\
H.R. Saibil & $80(30)$ & 4234 & 33 & $10.67 \pm 3.53$ & 4.70 & 3.89 & $11.0(8.68 \pm 0.09)$ \\
\hline
\end{tabular}

* Calculated from the highest $h$ value. Values in parentheses are the best-fit values from $h(t)$ plots in the entire $t$ range

Table 2 Citation parameters for 12 astronomers selected by Abt (2011)

\begin{tabular}{llllllll}
\hline Author & $N(t)$ & \multicolumn{1}{l}{$L$} & \multicolumn{1}{l}{$l$} & \multicolumn{1}{l}{$a$} & $A$ & $10 b^{*}$ \\
\hline A & $592(47)$ & 58042 & 121 & $17.41 \pm 3.76$ & 26.28 & 3.96 & 25.74 \\
B & $540(50)$ & 36586 & 102 & $15.83 \pm 2.72$ & 14.63 & 3.52 & 20.4 \\
C & $721(50)$ & 26680 & 85 & $15.33 \pm 1.14$ & 10.67 & 3.69 & 17.0 \\
D & $715(46)$ & 36688 & 66 & $14.6 \pm 1.68$ & 17.34 & 8.42 & 14.35 \\
E & $510(50)$ & 14530 & 56 & $8.79 \pm 1.53$ & 5.81 & 4.63 & 11.2 \\
F & $109(50)$ & 7888 & 38 & $6.63 \pm 1.32$ & 3.16 & 5.46 & 7.6 \\
G & $251(59)$ & 6468 & 38 & $4.86 \pm 1.74$ & 1.86 & 4.48 & 6.44 \\
H & $363(59)$ & 6950 & 37 & $6.34 \pm 0.75$ & 2.00 & 5.08 & 6.27 \\
I & $182(46)$ & 3401 & 34 & $8.94 \pm 1.38$ & 1.61 & 2.94 & 7.39 \\
J & $220(65)$ & 3788 & 30 & $4.07 \pm 0.78$ & 0.90 & 4.21 & 4.62 \\
K & $111(43)$ & 1600 & 19 & $5.26 \pm 0.79$ & 0.87 & 4.43 & 4.42 \\
L & $155(49)$ & 1012 & 18 & $4.23 \pm 0.10$ & 0.42 & 3.12 & 3.67 \\
\hline
\end{tabular}

* Calculated from the highest $h$ value

citations, the $h$ index and the $\alpha$ index of different authors. In Table 2 the authors are listed by alphabetical letters arranged in decreasing order of peak $h$ indexes, whereas the publication duration $t$ of the authors was read off from the plots in the paper published by Abt (2011) of $h$ and $\alpha$ against the years $t$ following the publication of their first papers.

According to Hirsch (2005) the relationship between $L(t)$ and $h$ is given by

$$
L(t)=A h^{2}(t)
$$

where $A$ is an empirical constant, which Hirsch found to lie between 3 and 5. However, the value of the Hirsch constant $A$ for the authors of Tables 1 and 2 lies in a wider range between 2.9 and 32.7. According to the progressive nucleation mechanism cumulative citations $L_{\mathrm{sum}}(t)$ is related to the publication duration $t$ by (Sangwal 2012)

$$
L(t)=a t^{2},
$$

with the citation acceleration

$$
a=\frac{\lambda_{0} \cdot \Delta N}{\Theta_{0}},
$$

where $\lambda_{0}$ is a proportionality constant (unit: citations), $\Delta N$ is the average number of papers 
published per year (i.e. $\Delta N=N / t$; unit: papers/year) and $\Theta_{0}$ is a time constant (unit:year). Since the parameters $\lambda_{0}, \Delta N$ and $\Theta_{0}$ are independent of citation time $t$ and characterize the citation behaviour of an author, citation acceleration $a$ of an author is independent of publication duration $t$. The units of $a$ are citations/years ${ }^{2}$.

From Eqs. 1 and 2 one obtains the relationship between Hirsch index $h$ and publication duration $t$ of an author as

$$
h=b t,
$$

where $b$ is the slope of the plot of $h$ against $t$, given by

$$
b=\left(\frac{a}{A}\right)^{1 / 2} .
$$

The slope $b$ is a measure of the scientific activity of an author. The higher is the value of $b$, the higher is the activity of the author. The decade-based age-independent index $\alpha$ introduced by Abt (2011) is given by

$$
\alpha=\sum_{i=1}^{10}(\Delta h)_{i},
$$

where $(\Delta h)_{i}$ is the increase in $h$ index in the year $i$ of an author and the summation is carried out over a decade such that $1<i<10$. Obviously, the index $\alpha=10 b=10(a / A)^{1 / 2}$. Examples of the average values of $\alpha$ calculated by the present author for the scientists of Anderson et al. are shown in Fig. 1, whereas those for the astronomers in different decades are presented in the paper by Abt (2011).

From the average values of $\alpha$ in different decades for different authors, the average values of their "age-independent" index $\alpha$ in their entire citation careers were calculated. These values are listed in Tables 1 and 2 . The values of $10 \mathrm{~b}$ were calculated by using Eq. 4 with the final $h$ index and the citation period $t$ (i.e. by using Eq. 5 from the values of $a$ and A) and are given in Tables 1 and 2. In the last column of Table 1 are also included in the parentheses the best-fit values of $10 \mathrm{~b}$ obtained on the assumption of linear dependence between $h$ index and the citation period $t$ of the selected scientists of Anderson et al. (see Fig. 2). Note that the slope $b$ of the plot of $h$ against $t$ for an author in Fig. 2 usually does not remain constant in the entire career of an author. In fact, the dependence of $h$ on $t$ is close to linearity only for Proctor.

Fig. 1 Average values of $\alpha$ in different decades for the scientists of Anderson et al. (2008). The average values of $\alpha$ were calculated by the present author for the original data reported by Anderson et al. shown in Fig. 2

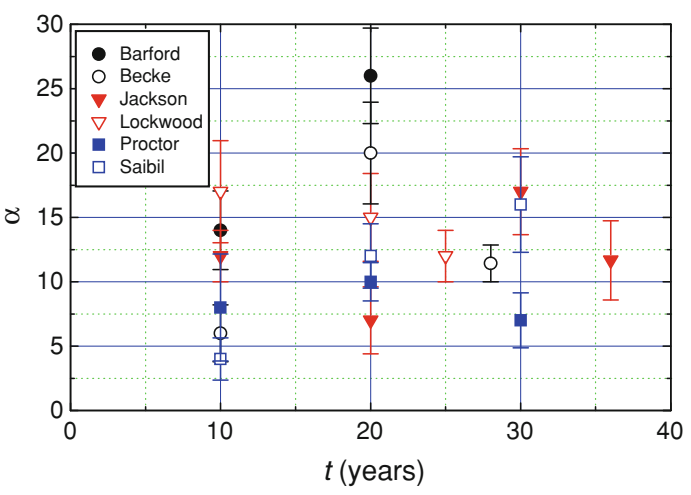


The relationship between age-independent index $\alpha$ and $10 b$ is shown in Figs. 3 and 4 for the selected "Royal" scientists of Anderson et al., and Abt's astronomers, respectively. In Fig. 3 the $\alpha$ index is plotted as a function of $10 b$ calculated in two ways: (a) from the highest values of $h$ index achieved in the scientific career $t$ using Eq. 4 and (b) from the linear dependence of $h$ on $t$. The solid line is drawn on the assumption that $\alpha=10 \mathrm{~b}$. In Fig. 4 the solid line presents the best-fit linear relationship between $\alpha$ and $10 b$, whereas the dashed line is drawn on the assumption that $\alpha=10 b$. It may be seen that in Fig. 3 the values of $\alpha$ are in excellent agreement with the values of $10 \mathrm{~b}$ calculated by the two methods. In contrast to Fig. 3, in Fig. 4, except for authors A and B, the values of $\alpha$ reported by Abt are comparable with the values of $10 b$ calculated by using Eq. 5. However, strictly spoken, the observed values of $\alpha$ by Abt are equal to $10 b$ for authors $\mathrm{D}, \mathrm{F}, \mathrm{H}, \mathrm{J}, \mathrm{K}$ and L, they are lower than the expected ones for authors A B, C, E and G, whereas the observed value of $\alpha$ is higher than the expected one for author I. These deviations in the observed values of $\alpha$ from the values expected from Eq. 5 are associated with different slopes of the plots of $h$ index on time $t$ in different decades of publications by these authors. In general, a pronounced increase in $h$ in the later stage of the scientific career of an author will lead to a higher value of his/her $\alpha$ whereas a decrease in $h$ in the later stage will lead to a lower value of $\alpha$. These features can be clearly seen in Fig. 3. The former feature may be observed in the case of Barford and Saibil whereas the latter feature is somewhat recognizable in the case of Becke.

Fig. 2 Relationship between $h$ index and the citation period $t$ of the selected scientists. Data are taken from Anderson et al. (2008). Only for Proctor the dependence of $h$ on $t$ is close to linearity

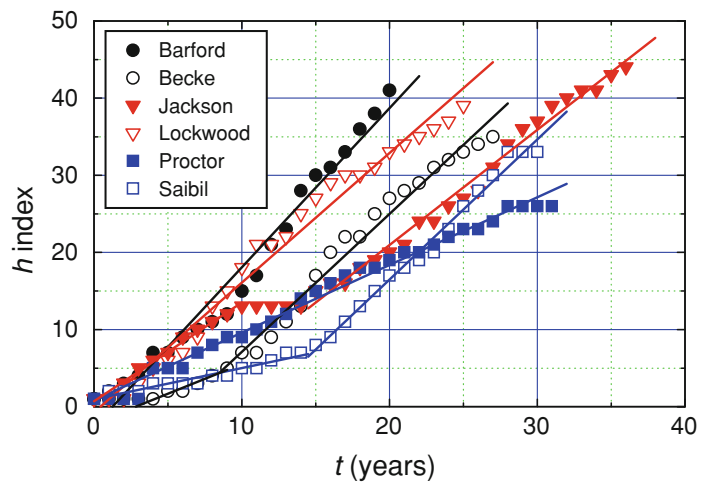

Fig. 3 Data of age-independent index $\alpha$ for the selected scientists of Anderson et al. plotted as a function of $10 \mathrm{~b}$ calculated in two ways: (open circles) from the the values of $a$ and $A$ given in column 8 of Table 1 using Eq. 5, and (filled circles) from the linear dependence of $h$ on $t$. Solid line is drawn on the assumption that $\alpha=10 b$

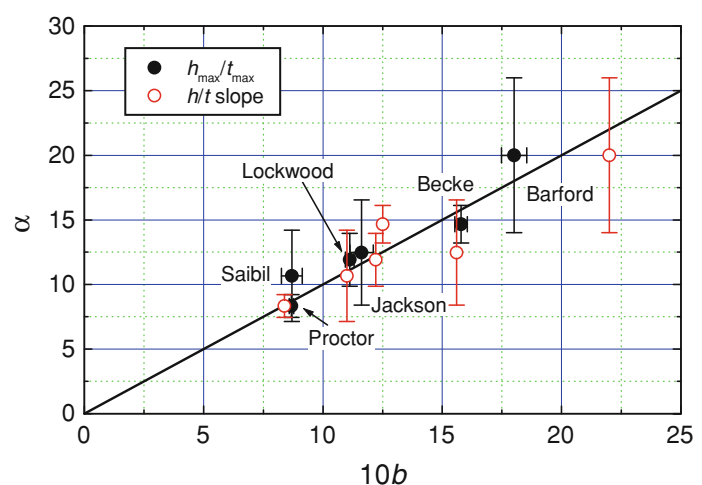


Fig. 4 Relationship between $\alpha$ given by $\mathrm{Abt}$ and $10 \mathrm{~b}$. Solid line presents the best-fit linear relationship between $\alpha$ and $10 b$ in the form: $\alpha=1.34+7.85 b$, with $r^{2}=0.978$. Dashed line is drawn on the assumption that $\alpha$ given by Eq. 6 is equal to $10 b$

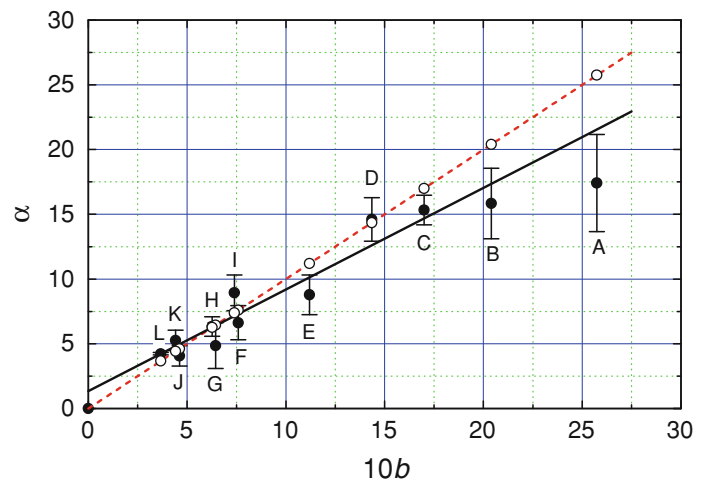

Fig. 5 Relationship between $\alpha$ and $a^{1 / 2}$ for different authors.

The linear relation is for Abt's astronomers and is: $\alpha=2.02+3.37 a^{1 / 2}$, with $r^{2}=0.962$

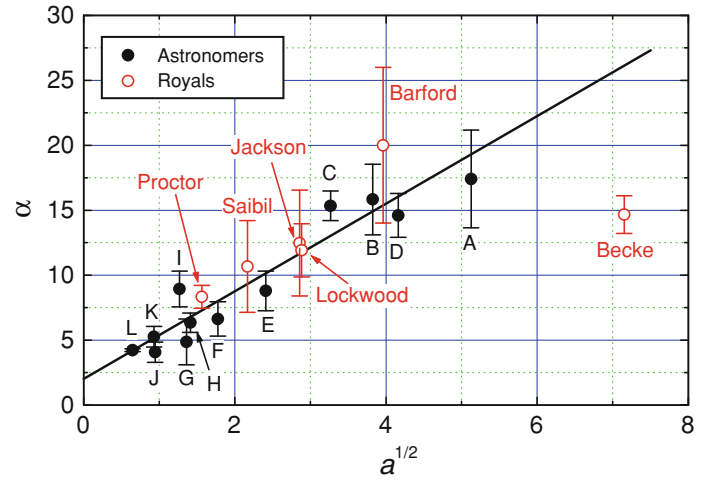

As seen from Tables 1 and 2, the Hirsch constant $A$ for a majority of scientists lies between 3 and 5.5. This means that one expects a linear relationship between $\alpha$ and $a^{1 / 2}$ (cf. Eq. 5). Figure 5 shows the data of $\alpha$ against $a^{1 / 2}$ for Abt's astronomers and scientists of Anderson et al. The straight line presents the best-fit plot of the $\alpha\left(a^{1 / 2}\right)$ data for Abt's astronomers. With the exception of Becke, one finds that the $\alpha\left(a^{1 / 2}\right)$ data for other "Royal" scientists are also described by this linear plot and the fit is similar to that observed in Fig. 4. This suggests that that citation acceleration $a$ is also a convenient measure to compare the publication output of different authors. Moreover, in comparison with the Hirsch $h$ index or age-independent $\alpha$ index, it is relatively easy to compute $a$ from the cumulative citations $L(t)$ after time $t$ (see Eq. 2).

Open Access This article is distributed under the terms of the Creative Commons Attribution Noncommercial License which permits any noncommercial use, distribution, and reproduction in any medium, provided the original author(s) and source are credited.

\section{References}

Abt, H. A. (2011). A publication index that is independent of age. Scientometrics. doi: 10.1007/s11192011-0525-4.

Anderson, T. R., Hankin, R. K. S., \& Killworth, P. D. (2008). Beyond the Durfee square: Enhancing the h-index to score total publication output. Scientometrics, 76, 577-588. 
Hirsch, J. E. (2005). An index to quantify an individual's scientific research output. Proceedings of the National Academy of Sciences of the USA, 102, 16569-16572.

Kosmulski, M. (2009). New seniority-independent Hirsh-type index. Journal of Informetrics, 3, 341-347.

Sangwal, K. (2012). On the time dependence of growth of citations of cumulative papers of individual authors and the concept of citation force $F$, Journal of Information Science, submitted for publication. 